\title{
ANTIFUNGAL PROPERTIES OF CHITOSAN MICROCAPSULES CONTAINING CINNAMON OIL AND LEMONGRASS OIL AGAINST ASPERGILLUS FLAVUS ISOLATED FROM STORED RICE
}

\author{
Yashodha L. Paragodaarachchi ${ }^{\mathrm{a}}$, Prarthana Subasinghe ${ }^{\mathrm{b}}$ and \\ Suranga R. Wickramarachchi ${ }^{\mathrm{a}^{*}}$
}

${ }^{a}$ Department of Chemistry, University of Kelaniya, Dalugama, Kelaniya, Sri Lanka

${ }^{b}$ Postgraduate Institute of Science, P.O. Box 25, Peradeniya, Sri Lanka

\begin{abstract}
Essential oils have long been attracted as substitutes for synthetic chemical pesticides because of their antimicrobial properties, low mammalian toxicity and wide public acceptance. Essential oils of lemongrass and cinnamon leaf were encapsulated using chitosan, a biodegradable polymer by emulsion formation/ionotropic gelation technique. A. flavus was isolated from stored rice. In-vitro antifungal efficacy of oil loaded microcapsules against $A$. flavus and its time profile were determined.

Based on morphological and molecular characteristics the fungus was identified as Aspergillus flavus. Both lemongrass and cinnamon leaf oil and their respective microcapsules were fungicidal against $A$. flavus. The Minimum inhibitory oil load of lemongrass and cinnamon leaf oil microcapsules were $0.5 \mathrm{~g}$ and $0.75 \mathrm{~g}$ respectively. Lemongrass showed a comparatively higher activity than cinnamon against the test fungi. However, cinnamon possessed the antifungal activity for a longer duration than lemongrass. Encapsulation of oil within chitosan may effectively reduce the evaporation rate of oil thus increasing its bioavailability. Microcapsules of cinnamon and lemongrass oil have the potential to be developed as a natural fungicidal formulation to control A. flavus.
\end{abstract}

\footnotetext{
*Suranga, Wickramarachchi, e-mail: suranga@kln.ac.lk
} 
Keywords: Microencapsulation, ionotropic gelation, lemongrass oil, cinnamon oil, Aspergillus flavus

\section{Introduction}

Botanical pesticides have long been attracted as substitutes for synthetic chemical pesticides due to increasing consumer concerns about pesticide residues on food, and threat to human health and environment. Essential oils are of huge interest in food applications because of their antimicrobial properties and low mammalian toxicity, less environmental effects and wide public acceptance. ${ }^{1}$ Oregano, thyme, clove, lavender, clary sage, and arborvitae essential oils have shown antifungal activity against Chaetomium globosum, Penicillium chrysogenum, Cladosporium cladosporoides, Alternaria alternata, and Aspergillus fumigatus. ${ }^{2}$ Cinnamon oil has shown antifungal activity against many fungi including Coriolus versicolor, Laetiporus sulphurius, Eutorium species, Aspergillus and Penicillium species. ${ }^{3}$ A study carried out in Sri Lanka to control anthracnose and crown rot in embul banana has shown the fungistatic activity of cinnamon and clove oil on Colletotrichum musae, Lasiodiplodia theobromae and Fusarium poliferatum. Lemongrass oil has been fungistatic and fungicidal against Aspergillus flavus in stored rice and also against Candida species. ${ }^{4,5}$

However, the direct application of the oil on the target organisms has many drawbacks. The sensitivity of oil to light, heat, moisture and oxygen prevents harnessing their full potential. Further, the direct application of oil on the food material contribute to unpleasant taste and aroma in food. Therefore, commercial application of these oils needs a formulation which can prevent above drawbacks and prolonging the bioavailability of the oil. Encapsulation is the most widely used and an efficient method by which 
solids, liquids or even gases may be enclosed in a polymeric microscopic particles of wall material. Encapsulation protects the oil from oxidation, isomerization and degradation while increasing the bioavailability of the core material as well as controls the delivery of functional substances. ${ }^{6}$

Several techniques are used in industrial controlled release formulations such as chemical binding of the polymerizing site to the active ingredient followed by polymerization of the new derivative, matrix encapsulation and microencapsulation. In microencapsulation, active material is coated with a thin film or a wall. The particles thus obtained are called microcapsules (MCs). The structure of the MCs varies. Some have a spherical geometry with a continuous shell. Others have an irregular geometry and contain a number of small droplets or particles of core material (Fig. 1). ${ }^{6}$

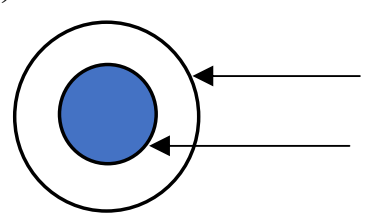

$1 \mathrm{~A}$

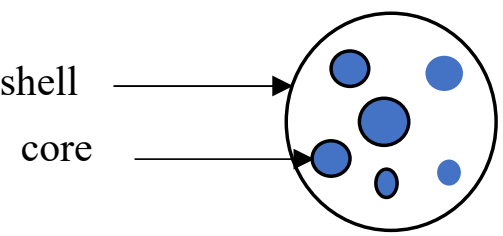

1B

Figure 1. Diverse types of MCs (A) continuous core/shell MC (B) multinuclear MCs.

Microencapsulation has been widely used to mask the odor and taste of many drugs to improve patient compliance. The liquid drugs can be converted into a powder and oxygen and heat sensitive drugs can be protected using microencapsulation. ${ }^{7}$ Encapsulation of essential oils have many applications in food, agriculture, pharmaceutical industry, textile an d footwear applications. ${ }^{6}$ Encapsulated marine and vegetable oils are used frequently in food industry because of the presence of omega-3 fatty acids in oil. ${ }^{8}$ By including microencapsulated antimicrobial agents within the fiber matrix, antimicrobial textiles are made. ${ }^{9}$ Mango fruits treated with 
encapsulated rosemary and garden thyme showed controlled decay, prolonged storage life, and the internal quality of the fruit was maintained. ${ }^{10}$ In Sri Lanka, studies on microencapsulation of cinnamon oil to improve its usability as a digestive supplement ${ }^{11}$ and impregnation of citronella oil microcapsules into cotton fabric as mosquito repellant clothes ${ }^{12}$ have been reported.

Fungal infestation contributes substantially to the postharvest losses of grain crops during storage, in Sri Lanka. It is estimated $5 \%$ of the total paddy production stored by farmers is lost during storage whereas $3 \%$ is lost at commercial storage partly due to fungal infestation. ${ }^{4}$ In the tropics Aspergillus spp. are more prominent due to their adaptation to dry environments. Aflatoxins are mycotoxins produced by A. flavus, A. parasiticus and $A$. nomius. A. flavus is one of the major storage fungi found regularly in agricultural commodities which produces aflatoxins such as aflatoxin $B_{1}, B_{2}, G_{1}, G_{2}$. The international agency for research on cancer has classified aflatoxins as human carcinogens. In tropical and Mediterranean climatic regions aflatoxins are generally formed after harvest under natural conditions. The presence of aflatoxigenic fungi and aflatoxins in paddy and maize $e^{4,13}$ grown in Sri Lanka has been reported. A previous study has revealed the levels of aflatoxins in rice are below the tolerance limit. ${ }^{4}$ However, continuous consumption of rice contaminated with aflatoxins even at low levels, could lead to adverse health effects.

The objective of this study is to determine the efficacy of lemongrass and cinnamon oil encapsulated (micro)formulations to control the stored grain pathogenic fungi, Aspergillus flavus under in-vitro conditions. To the authors knowledge any such microencapsulated formulation has not been evaluated to control stored grain fungi in Sri 
Lanka. As such this will be the first attempt in Sri Lanka to control a stored grain fungus using an encapsulated essential oil formulation.

\section{Materials and Method}

\section{Materials}

Chitosan (CS), medium molecular weight with viscosity of $36.5 \mathrm{cps}$ was purchased from Biotech Surinodo, Indonesia. Acetic acid was purchased from Sigma Aldrich (Molecular weight of $60.05 \mathrm{~g} / \mathrm{mol}$ with $99.5 \%$ assay). Tween 80 (Molecular weight of $604.822 \mathrm{~g} / \mathrm{mol}$, density of $1.06-1.10 \mathrm{~g} / \mathrm{mL}$ ) was used without further purification. Lemongrass oil (LGO) was purchased from Wrook infinite (PVT) Ltd., Sri Lanka and cinnamon oil (CNO) was purchased from the local market. All the chemicals used for DNA extraction were purchased from Sigma Aldrich.

\section{Method}

1. Formulation of Lemongrass oil containing Chitosan microcapsules (LGO-CS-MCs) and Cinnamon oil containing chitosan microcapsules (CNO-CS-MCs) using ionotropic gelation method

$1 \% \mathrm{CS}$ solution in $1 \%$ acetic acid $(\mathrm{w} / \mathrm{v})(100 \mathrm{~mL}$, ) was taken into a beaker. To this, oil (3g) was added dropwise with slow agitation (200 rpm) using an overhead stirrer (IKA RW 20 digital) at room temperature. Mixture was agitated using high speed $(1000 \mathrm{rpm})$ for 10 minutes to obtain a good emulsion. The speed was decreased to $200 \mathrm{rpm}$ and $0.5 \%$ sodium tripolyphosphate (STPP) was added dropwise until a good yield of MCs were observed under the microscope. Solution was stirred at the same speed for further 30 minutes. Microcapsules were centrifuged at high speed (6000 rpm) for 15 minutes. Supernatant was discarded, and the resulting 
pellet was washed with ethanol. MCs were freeze dried using the freeze dryer (LABONCO) and stored in the refrigerator until further use.

\section{Isolation of $A$. flavus from stored grain}

Stored grains (old rice sample) were soaked in sterile sodium hypochlorite (5\%) solution for $2 \mathrm{~min}$. for surface sterilization. Those grains were then rinsed with sterile distilled water and placed on a sterile paper towel inside bio safety cabinet for $2 \mathrm{~min}$. (to dry).The dried grains were placed on a petri-dish containing sterile Potato Dextrose Agar Medium (PDA) and the plates were incubated at ambient room temperature $\left(30 \pm 5^{\circ} \mathrm{C}\right)$ for five days.

3. Characterization and molecular identification of isolated A. flavus

Fungal colonies that grew on the incubated plates were sub-cultured onto fresh PDA plates and incubated to obtain pure cultures. The purified isolates were kept in PDA slants and stored in the culture collection at the Department of Chemistry, University of Kelaniya.

Colony morphology, and microscopic features of each fungal culture was observed under light microscope (ZEISS). Fungal colonies with putative morphological features as Aspergillus flavus were selected for molecular identification. Cultures were grown in Potato Dextrose Broth (PDB) for 7 days at room temperature and mycelial mat was used for DNA extraction. DNA extraction was done using CTAB method. ${ }^{14}$ Extracted DNA was subjected to Polymerase Chain Reaction (PCR) as described by Madhuranga et al. ${ }^{15}$ using ITS 1 and 4 universal primers. ${ }^{16} \mathrm{PCR}$ products were separated in agarose gel electrophoresis (Cleaver Scientific, UK). Pure single PCR products were sequenced using Macrogen sequencing 
technology at Genetech, Sri Lanka. Nucleotide sequences were manually edited using Bio Edit ver. and compared to the GenBank data base using Basic Local Alignment Search Tool (BLASTn). Obtained sequences were submitted to GenBank as a nucleotide query and compared with already existing DNA sequences using NCBI BLASTn ((http://www.ncbi.nlm.nid.gov/blast/)

4. In-vitro antifungal efficiency of LGO-CS-MCs and CNO-CSMCs

Antifungal activity of vapors of encapsulated essential oils was evaluated in-vitro by the method described by Soliman and et al ${ }^{17}$ with slight modifications. The antifungal assays were conducted in petri plates (90 mm diameter) of Potato Dextrose Agar (15 ml/plate) supplemented with chloramphenicol $(10 \mu \mathrm{g} / \mathrm{ml})$. Petri plates were inoculated evenly with $1 \mathrm{~mL}$ spore suspension (spore suspension was prepared by flooding sterile $1 \%(\mathrm{v} / \mathrm{v})$ saline solution $(10 \mathrm{ml})$ over a week-old pure culture. The hyphal fragments were removed by filtering through a sterile absorbent cotton wool plug). A sterile filter paper disk (30 mm diameter) was fixed in the center of the lid of the petri dish. Different amount of each oil $(0.1,0.25,0.50 \mathrm{~g})$ was distributed on to the sterile filter paper. Microcapsules were placed on the center of the lid using sterile solidification agar. The amounts of the oil encapsulated in the MCs were kept equivalent to the used free oil amounts $(0.1,0.25,0.50 \mathrm{~g})$. Sterile blank filter paper disk was considered as the control for the free oil and empty microcapsules $(0.50 \mathrm{~g})$ were used as the control for oil loaded MCs. The vertical distance between the surface of the growth media and the oil loaded filter paper disk or adhered MCs on the lid were kept constant $(5.6 \mathrm{~mm})$. Plates were sealed with parafilm and incubated at $28 \pm 2{ }^{\circ} \mathrm{C}$ for five days. Growth inhibition was determined by 
measuring the diameter of the inhibition zone and the percentage growth inhibition was calculated using the equation given below. The experimental design was a completely randomized design (CRD) with three replicates. Carbendazim (commercial fungicide) was used as the positive control.

Growth inhibition $(\%)=\frac{T-C}{90-C} \times 100$ where, $C=$ Mean radial diameter of the inhibition zone of the control plate $(\mathrm{mm})$. $T=$ Mean radial diameter of the inhibition zone of test plate $(\mathrm{mm})$.

Diameter of the petri plate $=90 \mathrm{~mm}$

5. Antifungal activity profile of free oil, LGO-CS-MCs and CNO-CS-MCs

Filter paper discs loaded with free oils $(0.5 \mathrm{~g})$, LGO-CS-MCs and CNO-CS-MCs (an equivalent amount of MCs containing $0.5 \mathrm{~g}$ of oil) were stored at room temperature $\left(28 \pm 2^{\circ} \mathrm{C}\right)$ for $2,4,7,10$ and 14 days. At the end of the storage period their antifungal activity was measured as described above in 2.5 .

\section{Results and Discussion}

\section{Formulation of MCs}

MCs were formulated using ionotropic gelation. During ionotropic gelation, phosphate groups of STPP form crosslinks between CS polymer strands by binding to the free protonated amine groups aggregating the dispersed CS strand (Fig. 2). 

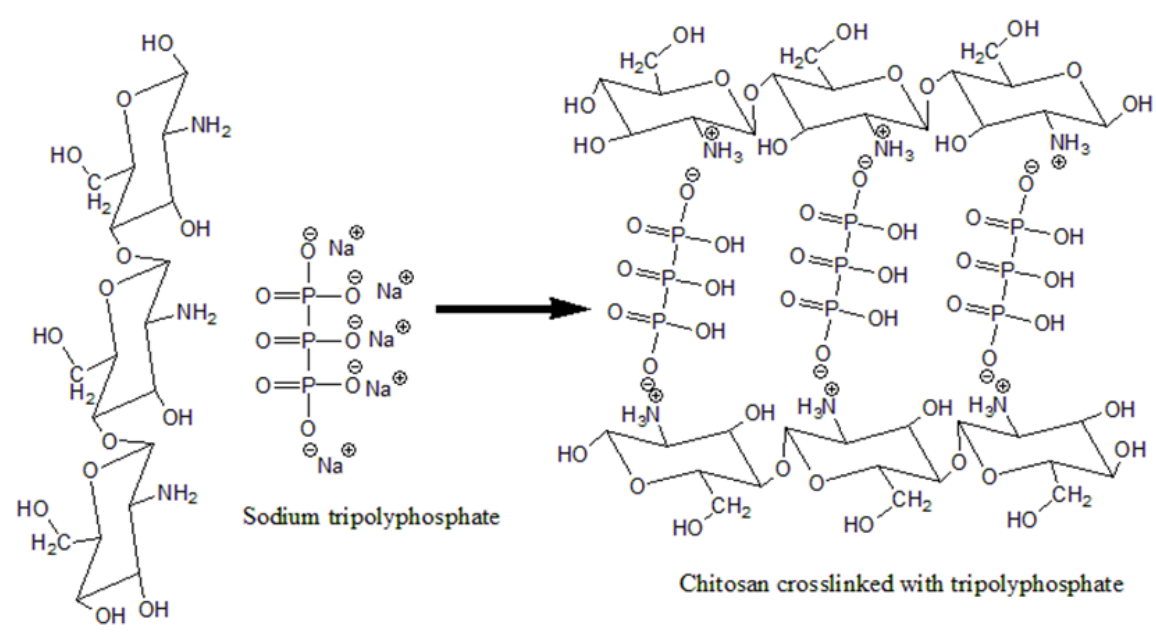

Chitosan

Figure 2. Interaction of CS with STPP (ionic crosslinking).

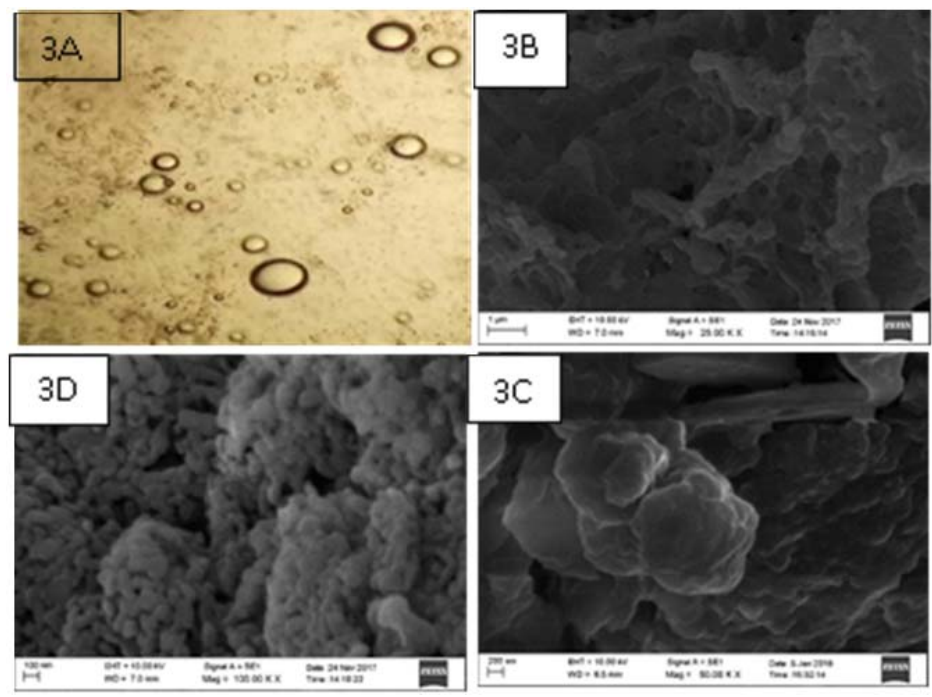

Figure 3. (A) Optical microscopic images of CNO-CS-MCs (magnification (10x100)), Scanning electron microscopic images of (B) CS-MCs (C) LGO-CS MC (D) CNO-CS-MC.

Authors have reported the formulation and characterization of $\mathrm{CNO}$ and LGO loaded MCs elsewhere. ${ }^{18,19}$ Briefly, SEM analysis shows that oil loaded MCs are spherical in shape (Fig. 3(C \& D)) with a particle size in the range $100-1300 \mathrm{~nm}$. FTIR confirms the successful encapsulation of oil within the wall material (Fig. 5). GC analysis of the two oils has shown the 
presence of citral-b (34.12\%) and citral-a (44.31\%) as the major constituents in $\mathrm{LGO}$ whereas in $\mathrm{CNO}$, eugenol $(76.9 \%)$ present as major constituents. Here we report the antifungal activity of CNO and LGO loaded MCs.

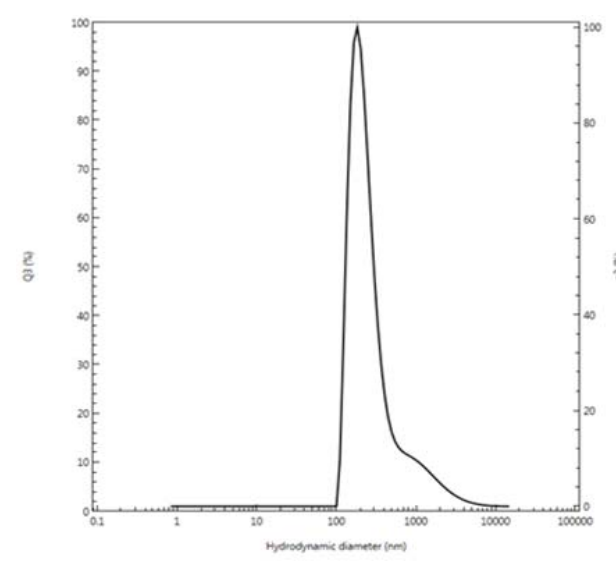

(4A)

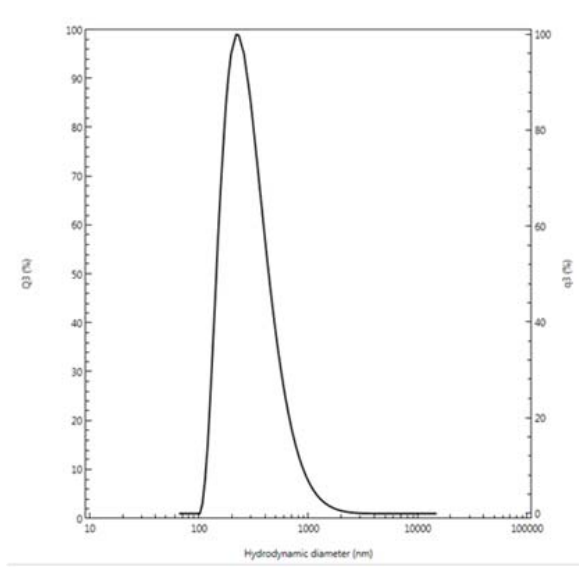

(4B)

Figure 4. Particle size distribution of dried (A) CNO-CS-MC ${ }^{19}$ and (B) LGO-CS-MC.

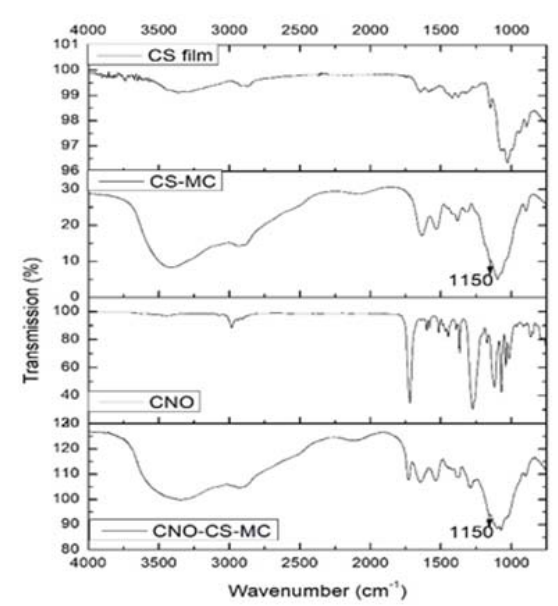

(5A)

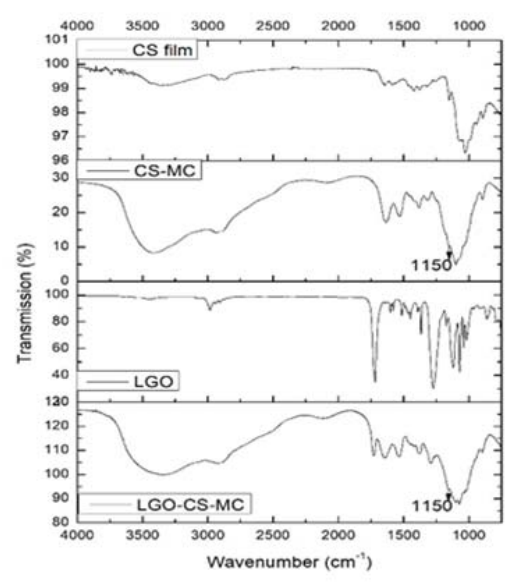

(5B)

Figure 5. FTIR spectra of pure oil, CS film, CS-MC and oil loaded MC (A) $\mathrm{CNO}^{19}$ (B) LGO. 


\section{Identification of $A$. flavus isolated from stored rice}

The fungus was identified based on morphological and molecular characteristics. Yellowish green color velvet-like smooth colony is observed on PDA. Conidia are light grey green, white powdery mycelium and exudates are present on surface (Fig. 6). The ITS sequence matches with A. flavus sequences from Genbank with $100 \%$ sequence identity hence the fungus was identified as Aspergillus flavus.

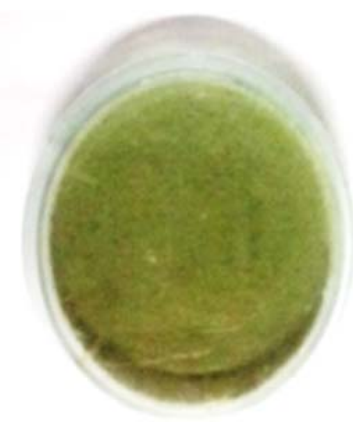

$(6 \mathrm{~A})$

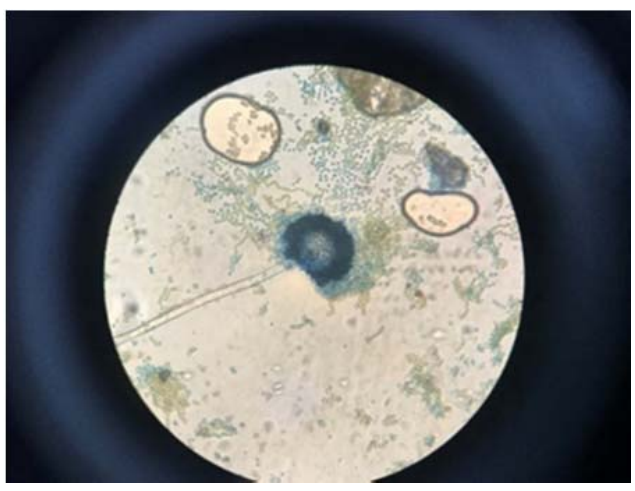

(6B)

Figure 6. A. flavus (A) pure culture plate (B) microscopic view.

\section{In-Vitro antifungal activity of oil loaded CS-MCs}

Antifungal activity was evaluated for different oil volumes $(0.1-$ $3.0 \mathrm{~g}$ ). Growth inhibition of A. flavus is dose dependent (Table 1 and Fig. 7). Both free oil and oil loaded MCs are fungistatic and fungicidal against $A$. flavus. Free LGO shows $100 \%$ inhibition of the fungus even at the lowest oil amount evaluated $(0.1 \mathrm{~g})$ whereas $\mathrm{CNO}$ shows fungicidal activity at $0.5 \mathrm{~g}$ of oil. Both CNO-CS-MC and LGO-CS-MC inhibit the growth of the test fungus. However, the antifungal activity of the oil is reduced after encapsulation thus proving the slow (controlled) release of the oil by the 
MCs. Here the active agent is released by diffusion through the micropores of the wall material (Fig. 8).

Table 1. Percentage inhibition of CNO, CNO-CS-MC, LGO and LGO-CS-MC.

\section{Amount of}

Inhibition (\%)

oil (g)

\section{CNO}

LGO

Free oil Oil loaded MCs Free oil Oil loaded MCs

$\begin{array}{lllll}0.10 & 59.25 \pm 3.90^{\mathrm{a}} & 39.63 \pm 0.65^{\mathrm{b}} & 100.00 \pm 0.00^{\mathrm{a}} & 54.81 \pm 1.28^{\mathrm{b}} \\ 0.25 & 80.37 \pm 2.80^{\mathrm{a}} & 53.33 \pm 2.94^{\mathrm{b}} & 100.00 \pm 0.00 & 97.03 \pm 3.40 \\ 0.50 & 100.00 \pm 0.00 & 80.00 \pm 2.23 & 100.00 \pm 0.00 & 100.00 \pm 0.00 \\ 0.75 & 100.00 \pm 0.00 & 100.00 \pm 0.00 & 100.00 \pm 0.00 & 100.00 \pm 0.00\end{array}$

$\begin{array}{lllll}\geq 1.00 & 100.00 \pm 0.00 & 100.00 \pm 0.00 & 100.00 \pm 0.00 & 100.00 \pm 0.00\end{array}$

* Mean of three replicates $(n=3)$. Data are presented as mean \pm standard deviation. Mean followed by different letters in superscript are significantly different from each other (ANOVA, Tukey's test, $\mathrm{p}<0.05$ ).

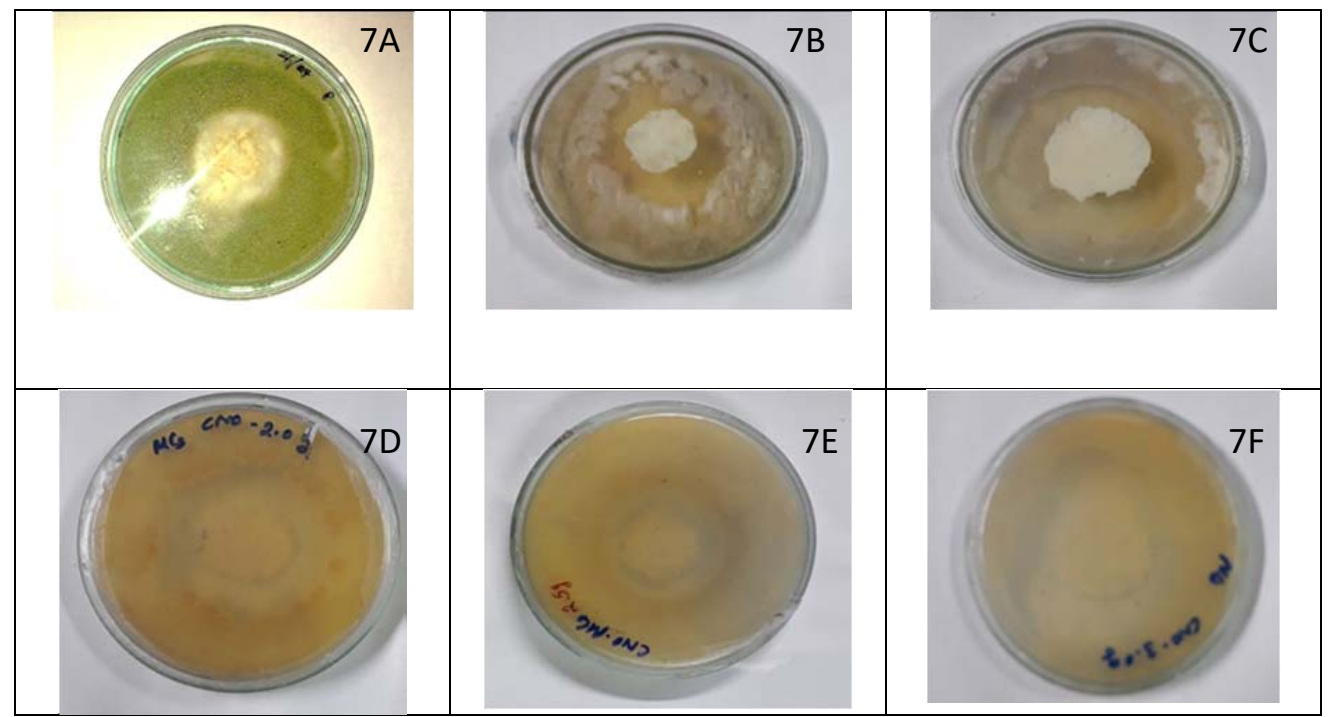

Figure 7. Inhibition zones of A. flavus treated with CNO-CS-MCs (A) 0.10 (B) 0.25 (C) 0.50 (D) 0.75 (E) 1.00 (F) $1.50 \mathrm{~g}$. 


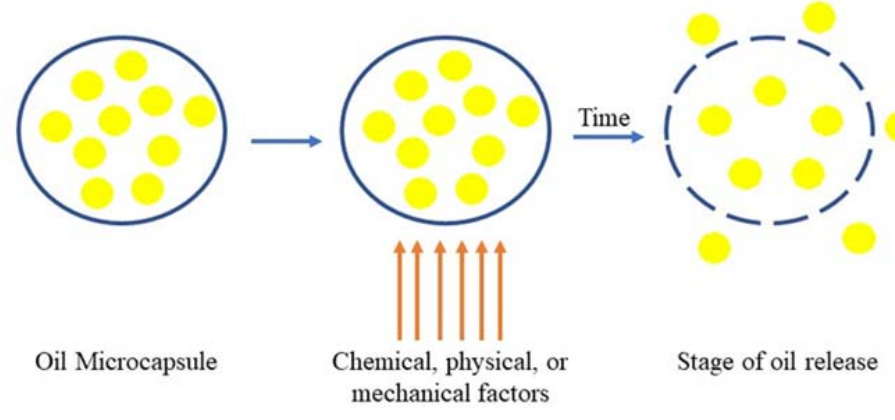

Figure 8. Schematic diagram of controlled release mechanism.

\section{Antifungal activity profile of free oil and oil loaded MCs}

The antifungal activity profiles of the free oil and oil loaded MCs with time are given in Table 2. Initially, both oils and oil loaded MCs show above $80 \%$ and $100 \%$ inhibition for CNO and LGO respectively. Within two days of storage both free oils lose their activity drastically and completly lose their effectiveness by seventh day. But, even after 07 days of storage, CNO-CS-MCs show above 55\% inhibition of the fungus while the LGO-CS-MCs show almost half of that of CNO-CS-MCs. Nevertheless LGO-CS-MCs still possess the activity compared to the free oil. After two weeks LGO-CS-MCs lose its activity while the CNO-CS-MCs still shows around $50 \%$ inhibition of the fungal growth. A similar trend is observed in the oil release profiles of LGO-CS-MCs and CNO-CS-MCs conducted under in vitro condition with a burst release of the oil at the initial stages and then slowly reaches to a plateau. ${ }^{18,19}$ These results thus prove that encapsulation prolongs the bioavailability of the oil. These results are supported by previous research. ${ }^{17}$ 
Table 2. Antifungal activity profiles of free oils and oil loaded MCs.

\section{Inhibition (\%)}

\section{CNO}

LGO

\section{Days CNO free oil CNO-CS-MC LGO free oil LGO-CS-MCs}

\begin{tabular}{ccccc}
\hline 0 & $90.37^{\mathrm{a}}$ & $84.07^{\mathrm{b}}$ & 100.00 & 100.00 \\
2 & $8.50^{\mathrm{a}}$ & $83.33^{\mathrm{b}}$ & $1.85^{\mathrm{a}}$ & $100.00^{\mathrm{b}}$ \\
4 & $1.67^{\mathrm{a}}$ & $61.66^{\mathrm{b}}$ & 0.00 & 95.18 \\
7 & 0.00 & 55.99 & 0.00 & 24.07 \\
10 & 0.00 & 49.63 & 0.00 & 16.66 \\
14 & 0.00 & 53.33 & 0.00 & 4.07
\end{tabular}

* Mean of three replicates $(n=3)$. Mean followed by different letters in superscript are significantly different from each other (ANOVA, Tukey's test, $\mathrm{p}<0.05)$.

During gelation, oil droplets are sealed inside the polymer coating by physical or chemical interactions of oils with the chitosan matrix. Previous studies have reported the formation of a reversible imine bond between primary amine groups and aldehydes. ${ }^{20,21}$ Aldehydes in LGO and CNO (citral, cinnamaldehyde, citronellal etc.) form reversible imine bonds with the amine group of chitosan. This is evidenced by the appearance of a new band at $1640 \mathrm{~cm}^{-1}$ for $\mathrm{C}=\mathrm{N}$ bond vibration and the decrease of the intensity of the $1540 \mathrm{~cm}^{-1}$ band for amine group vibration ${ }^{20}$ in the FTIR spectra of LGO-CS-MCs and CNO-CS-MCs (Fig 5A and 5B). However, this $\mathrm{C}=\mathrm{N}$ band is superimposed by the residual amide band at $1650 \mathrm{~cm}^{-1}$. The release of the oil occurs when the reversible imine bonds break due to physical, chemical or mechanical factors, and the oil diffuse through the pores left behind in the polymer coating (Fig. 8). Stanizone et $\mathrm{al}^{21}$ states that the release of the active compound is determined by its diffusion and the hydrolysis of the imine bond formed between the amino and aldehyde groups. 


\section{Conclusion}

The oil encapsulated MCs are fungicidal to A. flavus. LGO-CS-MCs is fungicidal at $0.5 \mathrm{~g}$ and $\mathrm{CNO}-\mathrm{CS}-\mathrm{MCs}$ is fungicidal at $0.75 \mathrm{~g}$. The fungicidal activity of LGO against A. flavus is higher than that of CNO in both free and encapsulated forms. However, the activity of CNO remains for a longer period than LGO. Both oils are potent fungicides of natural origin. Oil encapsulated form (as microcapsules) is more user friendly than the free oil in commercial applications.

\section{Acknowledgements}

Financial assistance by the University of Kelaniya research grant (RP/03/02/06/01/2016) is acknowledged.

\section{References}

1. Ntalli, N. G., Menkissoglu-Spiroudi U. Pesticides of Botanical Origin: A Promising Tool in Plant Protection, in M. Stoytcheva (Ed.), Pesticides Formulations, Effects, Fate, InTech: London, 2011, pp. 1-24.

2. Puškárová, A.; Bučková, M.; Kraková, L.; Pangallo, D.; Kozics, K. The antibacterial and antifungal activity of six essential oils and their cyto/genotoxicity to human HEL 12469 cells. Sci. Rep. 2017, 7, 1-11.

3. Mahmoud, A. L. E. Antifungal action and antiaflatoxigenic properties of some essential oil constituents. Lett. Appl. Microbiol. 1994, 19, 110113.

4. Paranagama, P. A.; Abeysekera, K. H. T.; Abeywickrama, K.; Nugaliyadde, L. Fungicidal and anti-aflatoxigenic effects of the essential oil of Cymbopogon citratus (DC.) Stapf. (lemongrass) against Aspergillus flavus Link. isolated from stored rice. Lett. Appl. Microbiol. 2003, 37, 86-90.

5. De Bona Da Silva, C.; Guterres, S. S.; Weisheimer, V.; Schapoval, E. E. S. Antifungal Activity of the Lemongrass Oil and Citral Against Candida spp. Brazilian. J. Infect. Dis. 2008, 12, 63-66. 
6. Bakry, A. M.; Abbas, S.; Ali, B.; Majeed, H.; Abouelwafa, M. Y.; Mousa, A.; Liang, L.; Microencapsulation of Oils: A Comprehensive Review of Benefits, Techniques, and Applications. Compr. Rev. Food. Sci. Food. Saf. 2016, 15, 143-182.

7. Ahirrao, S. P.; Gide. P. S.; Shrivastav. B.; Sharma, P. Ionotropic Gelation: A Promising Cross-Linking Technique for Hydrogels. J. Pharm. Nanotechnol. 2014, 2, 1-6.

8. Ye, A.; Cui, J.; Taneja, A.; Zhu, X.; Singh, H. Evaluation of processed cheese fortified with fish oil emulsion. Food Res. Int. 2009, 42, 10931098.

9. Özyildiz, F.; Karagönlü, S.; Basal, G.; Uzel, A.; Bayraktar, O. Microencapsulation of ozonated red pepper seed oil with antimicrobial activity and application to nonwoven fabric. Lett. Appl. Microbiol. 2013, 56, 168-179.

10. Kupaei, A.; Garmakhany, D. Effect of microencapsulated essential oils on storage life of mango fruit. Minerva Biotecnologica 2014, 26, 49-55.

11. Rasanganie, J. P. S.; Perera, B. G. K. Microencapsulation of cinnamon oil to improve its usability as a digestive supplement. Int. J. Sci. Res. Innov. Technol. 2018, 5, 130-144.

12. Wijayapala, S. Microencapsulation of Essential Oils (Citronella) and Impregnation into Cotton, Proceedings of the 23rd International Forestry and Environment Symposium, University of Sri Jayewardenepura, Sri Lanka, 2018, 119, 50.

13. Rajapakse, R.; Karunarathna, K.; Premarathne, P.; Perera, R. Evaluation of Maize Genotypes for Resistance to Aspergillus Infection and Aflatoxin Production. Trop. Agric. Res. Ext. 2011, 13, 68-72.

14. Cenis, J. L.; Rapid extraction of fungal DNA for PCR amplification. Nucleic Acids Res. 1992, 20, 2380.

15. Maduranga, K.; Attanayake, R. N.; Santhirasegaram, S.; Weerakoon, G.; Paranagama, P. A. Molecular phylogeny and bioprospecting of endolichenic fungi (elf) inhabiting in the lichens collected from a mangrove ecosystem in Sri Lanka. PLoS One 2018, 13, 1-22.

16. Attanayake, R. N.; Glawe, D. A.; McPhee, K. E.; Dugan, F. M.; Chen, W. Erysiphe trifolii - a newly recognized powdery mildew pathogen of pea. Plant Pathol. 2010, 59, 712-720. 
17. Soliman, E. A.; El-moghazy, A. Y.; El-din, M. S. M.; Massoud, M. A. Microencapsulation of Essential Oils within Alginate: Formulation and in Vitro Evaluation of Antifungal Activity. J. Encapsulation Adsorpt. Sci. 2013, 3, 48-55.

18. Paragodaarachchi, Y.; Wickramarachchi, S. Encapsulation of lemongrass oil in chitosan: formulation and characterization, Proceedings of the Forty Seventh Annual Sessions, Institute of Chemistry Ceylon, Sri Lanka, 2018, 35, 15-16.

19. Subasinghe, U. G. P. P.; Wickramarachchi, S. Encapsulation of cinnamon leaf oil within chitosan: formulation and characterization, Ceylon J. Sci. 2019, 48, 279-284.

20. Marin, L.; Simionescu, B.; Barboiu, M. Imino-chitosan biodynamers. Chem. Commun. 2012, 48, 8778-8780.

21. Stanzione, M.; Gargiulo, N.; Caputo, D.; Liguori, B.; Cerruti, P.; Amendola, E.; Lavorgna, M.; Buonocore, G. G. Peculiarities of vanillin release from amino-functionalized mesoporous silica embedded into biodegradable composites. Eur. Pol. J. 2017, 89, 88-100. 